\title{
Technique, complications, and clinical value of endomyocardial biopsy in patients with heterotopic heart transplants
}

\author{
DKC COOPER, RC FRASER, AG ROSE, O AYZENBERG, GS OLDFIELD, J HASSOULAS, D NOVITZKY, \\ CJ UYS, CN BARNARD
}

From the Departments of Cardiac Surgery, Cardiology, and Pathology, Groote Schuur Hospital and University of Cape Town Medical School, Cape Town, South Africa

ABSTRACT A review of 157 consecutive biopsies of donor endomyocardium in patients with heterotopic heart transplants is reported. The technique of percutaneous transvenous endomyocardial biopsy after this operation is described; manipulation of the catheter and bioptome into the junction of the donor superior vena cava and right atrium can be difficult when this anastomotic junction is small, as a result either of operative surgical technique or of subsequent contraction. The complication rate was $4 \%$ but one patient may have died from infection resulting from biopsy when the bioptome had to be introduced at the groin. The histopathological changes seen in the biopsy specimens have been graded according toa scoring system to give the clinician a guide to the severity of rejection. Histopathological assessment was of clinical value in $96 \%$ of cases, but was inaccurate on two occasions, once because an opinion was given on what was in retrospect an inadequate sample. In patients undergoing persistent low-grade acute or chronic rejection there was difficulty in detecting or appreciating the true extent of myocardial fibrosis; this led to inadequate immunosuppressive treatment in two patients. Attention is drawn to the fact that ischaemic fibrosis resulting from the vascular changes of chronic rejection may spare the endomyocardium, which is kept viable by intracavitary blood, and that this may lead to a misleading histopathological report.

The diagnosis of rejection in transplanted hearts, whether orthotopic or heterotopic, may be difficult and cannot await the appearance of clinical signs if severe immunological damage is to be avoided. Electrocardiographic changes may indicate rejection; a decrease in QRS voltage of $20 \%$ or more has long been held to suggest rejection, ${ }^{12}$ although there are many other factors which may influence the $\mathrm{QRS}$ voltage. ${ }^{3}$

Percutaneous transvenous or transarterial biopsy of right and left ventricles with a specially designed forceps, to provide fragments of endomyocardium for histological examination, was first described by Sakakibara and Konno in $1962^{4}$ and 10 years later was introduced as a clinical tool in heart transplantation by Caves and his colleagues. ${ }^{5}$ Biopsy remains the single most useful diagnostic aid in assessing rejection in the donor heart, being superior to electrocardiography ${ }^{67} ;$ since 1976 it has been used increasingly at Groote Schuur Hospital in the

Address for reprint requests: Mr DKC Cooper, Department of Cardiac Surgery, Groote Schuur Hospital, Cape Town 7925, Republic of South Africa. management of patients undergoing heart transplantation.

We have reviewed 157 consecutive biopsies of donor hearts performed from January 1979 to April 1981. In this report we emphasise the technical problems of biopsy in patients with heterotopicheart transplants, the complications of the procedure in our hands and the clinical value of the histopathological information obtained.

\section{Methods}

\section{THE PATIENTS}

Since 1979 it has been our policy to perform biopsies on donor hearts about once a week during the first month after transplantation, with decreasing frequency during subsequent months. Biopsy is also performed whenever a rejection episode is suspected, and toassess the efficacy of a course of anti-rejection treatment. From 1 January 1979 to 20 April 198116 heterotopic heart transplantation operations were performed, two of these being retransplantation procedures. During this period 157 


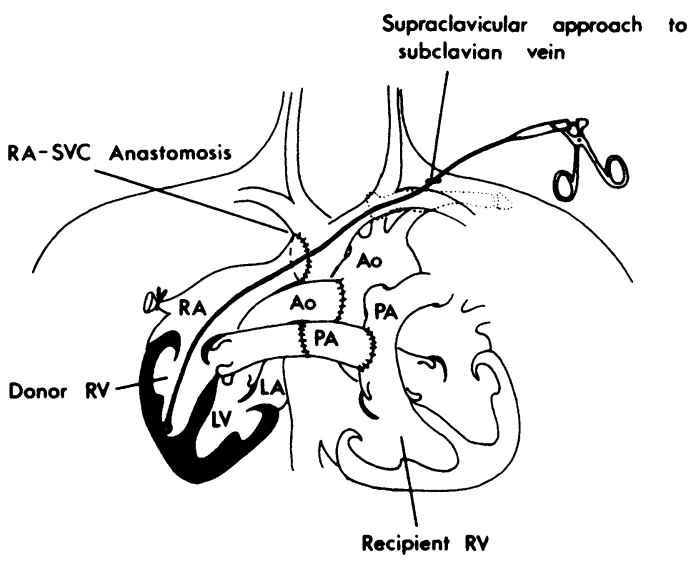

Fig 1 Diagram showing anatomical relationship of donor and recipient heart and passage of bioptome. $R A, L A-$ right and left atrium; $R V, L V$-right and left ventricle; $S V C$ - superior vena cava; Ao-aorta; $P A$ - pulmonary artery.

endomyocardial biopsy specimens were taken from a total of 19 patients. The number of biopsy specimens per patient ranged from one to 26 , eight patients undergoing more than 10 biopsy procedures each. On nine occasions full or limited left and right heart catheterisation studies with or without angiography were performed at the same time.

Cefamandole nafate (Mandokef - Lilly Laboratories SA (Pty) Ltd) $1 \mathrm{~g}$ is given intravenously one hour before biopsy and thereafter six hourly for 24 hours. Biopsy is not performed if the prothrombin index is below the therapeutic range.

\section{BIOPSY TECHNIQUE}

The percutaneous supraclavicular approach to either the right or the left subclavian vein is our preferred approach, though the infraclavicular route has been used in several patients. The procedure takes 15-30 minutes and is performed in the catheterisation laboratory. Once the subclavian vein has been entered, a 9-F Kifa catheter is passed via a Desilets-Hoffman sheath to the superior vena cava and manipulated through the anastomosis between donor and recipient superior venae cavae and right atria and across the donor tricuspid valve, and stabilised in the donor right ventricular cavity. The bioptome is then passed through the catheter (fig 1) so that multiple biopsy specimens can be obtained. Until recently an FB1C Olympus bioptome has been used; currently an Olympus 19C bioptome with fenestrated forceps is preferred as larger specimens may be obtained with this instrument. In patients with heterotopic heart transplants an appropriately sized J-type guide wire is a valuable aid if difficulty is encountered in manipulating the catheter across the anastomosis between the superior vena cava and the right atrium.

\section{HISTOLOGY}

The endomyocardial samples usually comprise one to four fragments, each 1-2 mm in diameter, submitted in $5 \%$ glutaraldehyde to facilitate subsequent ultrastructural examination of one of the fragments. Two fragments are subsequently fixed in $5 \%$ buffered formaldehyde and processed in the routine manner. Paraffinembedded sections are then stained by the haematoxylin-eosin, elastic van Gieson, and UnnaPappenheim methods.

Assessment of rejection is based on light microscopy; we have attempted to grade the histopathological changes seen, the aim being to give the clinician a guide to the severity of rejection and to the efficacy of antirejection treatment. The reproducibility of histological interpretation is enhanced since we always use the same criteria to assess the biopsy specimens and give them scores, rather than basing the diagnosis of rejection on an overall impression of the specimens.

Five histological criteria are independently assessed: interstitial oedema, interstitial mononuclear cell infiltration, cytoplasmic pyroninophilia of the mononuclear cells (as assessed on Unna-Pappenheim staining), myofibre degeneration, and blood vessel alterations. Myofibre alterations include oedema, loss of sharp contour, indistinct cross-striations, myocytolysis, fragmentation, and coagulative necrosis. Blood vessel alterations include intimal cell proliferation or necrosis, medial cell loss, and mononuclear infiltration of the vessel wall.

The presence or absence of each of these five histological criteria is scored from 0 to 3 as follows: 0 -absent or normal, 1-mild change, 2-moderate change, 3 severe change. The sum of these scores from the biopsy specimen showing the most severe changes of rejection is the final score and theoretically this may be as high as 15 , though in clinical specimens scores above 7 are rare (in the experimental animal, when acute rejection is allowed to progress to a conclusion, a score of 15 is not uncommon). In our experience a final score of 0 implies that no rejection is occurring, $0.5-2$ denotes mild acute rejection, 2.5-4 moderate acute rejection, and more than 4 severe acute rejection.

\section{Results}

Persistent difficulty was encountered in four patients, three immediately after operation and one three months after surgery. This was related to stenosis of the anastomosis, resulting from either operative technique or subsequent shrinkage during healing, or both. In three cases passage of the catheter from the right or left femoral vein overcame this problem. In one of these patients, however, the anastomosis contracted over nine months to a size where it became impossible to enter the donor right atrium at all. In such patients percutaneous trans- 
arterial biopsy of the left ventricle is necessary. Patients with a restrictive anastomosis of the superior venae cavae rely almost entirely therefore on their own right ventricle to support the pulmonary circulation, though the left ventricle of the transplanted heart continues to support the systemic circulation. No circulatory embarrassment has been observed, though dysrhythmias of both hearts occurred in one patient.

On three occasions (2\%) the procedure was abandoned without biopsy specimens, though in two cases success was achieved by a different route on a subsequent occasion. Failure to obtain specimens sufficient for histopathological studies has occurred on seven occasions (4.5\%) and has been most frequent in patients undergoing severe rejection resulting in poor myocardial function with subsequent ventricular mural thrombus formation. Adipose tissue only has been obtained on two occasions, but there were no clinical features to suggest that this was epicardial fat. Occasionally scanty chordal or valvular tissue, or both, has been obtained in addition to myocardial fragments.

\section{COMPLICATIONS}

One hundred and fifty-one biopsy procedures (96\%) were performed without complication. Repeated biopsy does not increase the risk - one patient underwent 26 biopsies without complications. Six complications occurred in all: one pneumothorax, two cases of transient nerve paresis induced by the anaesthetic (one a recurrent laryngeal paresis and one a partial brachial plexus paresis), one minor haemorrhage from the femoral artery, and two cases of possible infection. The pneumothorax required intercostal tube drainage. The femoral artery haemorrhage was controlled by a pressure bandage, and the nerve pareses resolved spontaneously in a few hours.

Two patients, both of whom required femoral artery cannulation for left ventricular biopsy, developed complications possibly due to infection, though these were not proved to have resulted from the biopsy procedure. One patient developed a pyrexia after biopsy that persisted for four days. Blood cultures gave negative results and no other features of infection were present. Cefamandole administration was extended to a total of six days and there was no further complication. The second patient developed a sterile, serous discharge at the site of insertion of the catheter three days after biopsy. Some four days later he developed bronchopneumonia with pyrexia, rigors, and sweating. Blood cultures grew Escherichia coli, but no organism was cultured from his sputum during life. Despite intensive antibiotic treatment he died from fulminant bilateral pneumonia, confirmed at necropsy, after which three organisms were cultured (E coli, Enterobacteraerogenes, and Peptococcus sp). Whether the endomyocardial biopsy caused the initial bacteraemia and this resulted in pneumonia or pneumonia caused the bacteraemia remains uncertain.
CLINICAL VALUE OF HISTOPATHOLOGICAL ASSESSMENT The scores given to the 157 biopsy specimens forming the basis of this paper are shown in table 1. In addition, fibrosis, suggesting previous myofibre damage from acute or chronic rejection, was seen in 42 samples.

Histopathological assessment was of clinical value in $96 \%$ of the cases in which the biopsy was adequate, but was interpreted incorrectly in clinical terms on four occasions and was misleading on two. The assessment was of value in either confirming or excluding the presence of suspected acute rejection or in showing its presence when it had not been suspected (table 2). The histopathological response to a more intensive course of immunosuppressive treatment could also be assessed as either indicating or ruling out the need for continued high-dose treatment; excessive treatment could thus be avoided.

It is difficult to be certain whether there have been any "false-positive" reports, though this would seem unlikely as a previous study from our institution showed that the histological changes of rejection are equally distributed between the left and right ventricles, including the interventricular septum. ${ }^{8}$ On occasion, within the 18-24 hours required to process and stain the biopsy tissue, electrocardiographic evidence of rejection has become manifest, supporting a positive biopsy result. In our experience the ECG voltage may be unchanged even with severe acute rejection and we have seen one patient with a biopsy score of 7.0 whose $Q R S$ voltage did not fall until the day after the biopsy.

Table 1 Histopathological scores for acute rejection in 157 endomyocardial biopsies

\begin{tabular}{lc}
\hline Degree of rejection & No (\%) of biopsies \\
\hline None (score 0) & $20(13)$ \\
Mild $(0 \cdot 5-20)$ & $57(36)$ \\
Moderate $(2 \cdot 5-4 \cdot 0)$ & $45(29)$ \\
Severe $(>4 \cdot 0)$ & $25(16)$ \\
Failure to obtain specimen & $3(2)$ \\
Inadequate tissue obtained & $7(4)$ \\
\hline Total & $157(100)$ \\
\hline
\end{tabular}

Table 2 Clinical value of histopathological assessment of specimens in 147 adequate endomyocardial biopsies

\begin{tabular}{lc}
\hline Result & No (\%) of biopsies \\
\hline Confirmed clinically suspected rejection & $25(17)$ \\
Excluded clinically suspected rejection & $15(10)$ \\
Demonstrated clinically unsuspected rejection & $25(17)$ \\
Confirmed clinical absence of significant & $47(32)$ \\
rejection & $29(20)$ \\
Showed expected response to increased & $4(3)$ \\
treatment & $2(1)$ \\
Report inadequately interpreted clinically & $147(100)$ \\
\hline Total &
\end{tabular}


If a rejection episode is strongly suspected - for example, on clinical or electrocardiographic grounds but is not confirmed by biopsy, the biopsy is usually repeated. In view of the subsequent clinical course and information from serial biopsies, we believe that on six occasions the information obtained from the biopsy specimens or their interpretation was or could have been misleading. The two causes of error have been an inadequate biopsy specimen on one occasion (on which we can see in retrospect that no report should have been proferred) and observer error on the part of the pathologist on the second, too low a score being given in both cases. On the four occasions on which interpretation may have been at fault the circumstances were similar and appear to indicate a need to remain suspicious of a "negative" histopathological report when there are strong electrocardiographic or other indications that rejection is occurring or that the donor heart function is deteriorating appreciably. Low scores were reported on four occasions (in two patients) when rejection was clinically suspected, on three occasions associated with evidence of impaired donor heart function. Both patients subsequently suffered irreversible loss of graft function. In each patient persistence or repeated episodes of relatively low-grade acute rejection or chronic rejection (immunologically induced accelerated coronary atherosclerosis), or both, over several weeks resulted in considerable replacement fibrosis. Although patchy fibrosis was observed in the biopsy specimen and reported, clinical attention was concentrated on the histological features of acute rejection and, as these were relatively minor, no extra treatment was administered. When chronic rejection increases there is little extra treatment that can be offered to the patient, but when the fibrosis is a result of repeated episodes of low-grade acute rejection then failure to increase treatment may lead to early graft failure. We describe one such case.

One year after transplantation, with only one moderate rejection episode during that period, the patient developed an appreciable and rapid fall in ECG voltage; at biopsy a score of 1.0 was obtained, and he was discharged from hospital and allowed to go on holiday. He returned a month later with graft failure, which proved irreversible even with massive and prolonged anti-rejection treatment. Even at this time, however, the features of acute rejection on histological examination were only moderate (score 3.5), though significant fibrosis was noted. It would seem likely that relatively lowgrade, untreated acute rejection persisting over one month, possibly combined with chronic rejection, led to severe myocardial damage and replacement fibrosis. The initial fall in ECG voltage, despite a histopathological report suggesting only mild acute rejection, should have been viewed more suspiciously and the patient followed up at an early date. Increased treatment at this stage may have averted graft failure, though if chronic

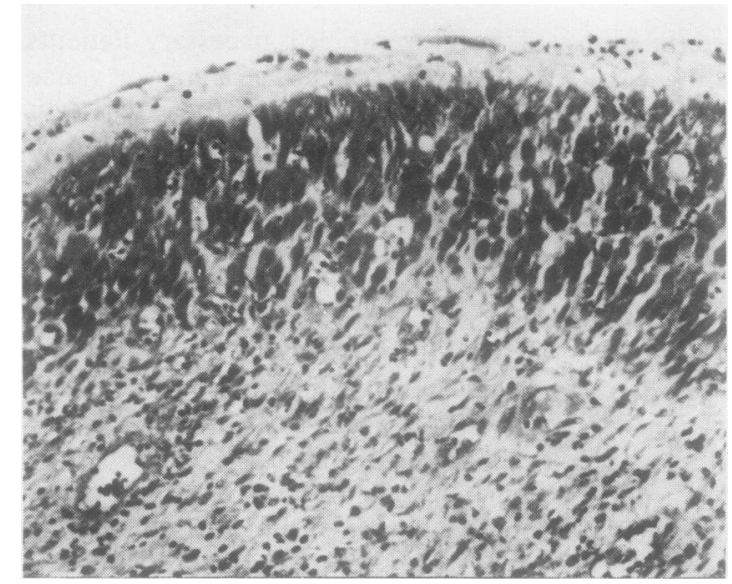

Fig 2 Section of myocardium from an allograft excised after cessation of function due to chronic rejection, showing widespread fibrosis but with relative sparing of the endomyocardium (top) (haematoxylin and eosin, $\times 110$ ).

rather than low-grade acute rejection was the main process no form of treatment is likely to have been successful. This patient has subsequently undergone excision of the donor heart and retransplantation.

Changes of chronic rejection are not always evident in the endomyocardium as the impairment of myocardial blood supply that results from immunologically.induced coronary vessel wall damage may spare the endomyocardium (fig 2), since this receives an adequate alternative blood supply direct from the ventricular cavity. This phenomenon may in part explain a histopathological report that does not reflect the true situation.

\section{Discussion}

Endomyocardial biopsy in heterotopic cardiac transplantation is a relatively safe invasive procedure with few important technical problems. About $94 \%$ of biopsy procedures provide tissue samples adequate for meaningful histopathological assessment.

Cefamandole given intravenously for 24 hours to cover the biopsy procedure was effective as prophylaxis against infection, with two possible exceptions-both of which were associated with circulatory access via the femoral vessels. Percutaneous transvenous endomyocardial biopsy performed by the subclavian route has not been associated with any infective complication in our hands.

Potential complications of this technique, not encountered in this series, include pulmonary embolism (or systemic embolism in the case of left ventricular biopsy) from dislodgement of mural stasis thrombus, which may result from decreased myocardial contractions due to rejection, and perforation of the right ventricular myocardium. (The latter has been a complication of one subsequent biopsy, $550 \mathrm{ml}$ of blood being 
aspirated from the right chest; the patient recovered fully.) The complication rate of the procedure, however, is said to be lower than that of liver or renal biopsy. ${ }^{9} \mathrm{Ven}$ tricular ectopic activity induced by the catheter or bioptome has not been a problem in this series.

The histological assessment was of clinical value in $96 \%$ of cases in which an adequate biopsy specimen was obtained, either confirming clinical or electrocardiographic suspicion of acute rejection or indicating rejection when it had hitherto been unsuspected. A biopsy providing no evidence of rejection is also of value as it prevents the needless continuation of a high-dose immunosuppressive regimen.

Occasional or even repeated biopsies may be misleading owing to difficulty in obtaining adequate tissue, observer error, incorrect interpretation of the histopathological features, or inadequate clinical follow-up in suspicious cases. In chronic rejection endomyocardial biopsy is less reliable since there is little chance of obtaining a small artery of the size affected by this process. In addition, the ischaemic fibrosis resulting from the vascular changes of chronic rejection may spare the endomyocardium, which is kept viable by the intracavitary blood. A "negative" histopathological report must therefore be viewed with suspicion when there are strong electrocardiographic or other indications that rejection is occuring.

Limited right or left ventricular angiography can be of great value in assessing donor heart function, gross angiographic dysfunction throwing suspicion on the histopathological assessment if a low rejection score is reported for biopsy specimens obtained at the same time. Serial monitoring of external (carotid or femoral) pulse traces can give similar information on graft function; both donor and recipient pulse waveforms can be recorded and their relative height and configuration may be helpful in showing a reduction in donor heart function, as occurs in severe rejection. Anti-rejection treatment should be instituted or continued despite repeatedly low histological scores if other features strongly suggest continuing rejection.
Endomyocardial biopsy remains, however, the single most useful indicator of graft rejection. The information it provides can be obtained with a relatively low complication rate (4\% in the present series) and with little discomfort to the patient. One patient, however, may have died from infection introduced at biopsy, and therefore the search for a non-invasive method of confirming, or preferably predicting, rejection continues.

We wish to thank the many other members of the medical, nursing, and laboratory staff of Groote Schuur Hospital and the University of Cape Town Medical School who have contributed to the care of patients undergoing heterotopic heart transplantation.

\section{References}

${ }^{1}$ Lower RR, Dong E Jun, Glazener FS. Electrocardiograms of dogs with heart homografts. Circulation 1966;33:455-60.

${ }^{2}$ Scheuer J, Shaver JA, Harris BC, Leonard JJ, Bahnson HE. Electrocardiographic findings in cardiac transplantation. Circulation 1969;40:289-96.

3 Baumgartner WA, Reitz BA, Oyer PE, Stinson EB, Shumway NE. Cardiac homotransplantation. Curr Probl Surg 1979;16:1-61.

${ }^{4}$ Sakakibara S, Konno S. Endomyocardial biopsy. Jpn Heart J 1962;3:537-42.

${ }^{5}$ Caves PK, Stinson EB, Graham AF, Billingham ME, Grehl TM, Shumway NE. Percutaneous transvenous endomyocardial biopsy. JAMA 1973;225:288-91.

${ }^{6}$ Caves PK, Billingham ME, Stinson EB, Shumway NE. Serial transvenous biopsy of the transplanted human heart-improved management of acute rejection episodes. Lancet 1974;i:821-6.

${ }^{7}$ Losman JG, Rose AG, Barnard CN. Myocardial fibrinolytic activity in allogenic cardiac rejection. Transplantation 1977;23:414-22.

${ }^{8}$ Rose AG, Uys CJ, Losman JG, Barnard CN. Evaluation of endomyocardial biopsy in the diagnosis of cardiac rejection. Transplantation 1978;26:10-13.

${ }^{9}$ Mason JW, Billingham ME, Rider AK, Harrison DC. Myocardial biopsy. In: Willerson JT, Sanders CA, eds. Clinical cardiology. New York: Grune and Stratton 1977:606-13. 